\title{
Curcumin plays a synergistic role in combination with HSV- TK/GCV in inhibiting growth of murine B16 melanoma cells and melanoma xenografts
}

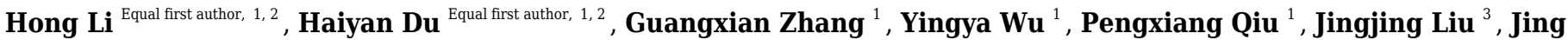 \\ Guo $^{3}$, Xijuan Liu ${ }^{1}$, Lingling Sun ${ }^{4}$, Biaoyan Du ${ }^{\text {Corresp., }{ }^{2,3} \text {, Yuhui Tan }}{ }^{\text {Corresp. } 1,2}$ \\ ${ }^{1}$ Department of Biochemistry and Molecular Biology, Guangzhou University of Chinese Medicine, Guangzhou, China \\ 2 The Research Center of Basic Integrative Medicine, Guangzhou University of Chinese Medicine, Guangzhou, China \\ 3 Department of Pathology, Guangzhou University of Chinese Medicine, Guangzhou, China \\ 4 Integrative Cancer Center, First Affiliated Hospital, Guangzhou University of Chinese Medicine, Guangzhou, China \\ Corresponding Authors: Biaoyan Du, Yuhui Tan \\ Email address: dubiaoyan@gzucm.edu.cn, yhuitan@foxmail.com
}

Melanoma is a global concern and accounts for the major mortality of skin cancers. Herpes simplex virus thymidine kinase gene with ganciclovir ( HSV-TK/GCV) is a promising gene therapy for melanoma. Despite its low efficiency, it is well known for its bystander effect which is mainly mediated by gap junction. In this study, we found that curcumin reduced B16 melanoma cell viability in both time- and dose-dependent manner. Further study showed that curcumin improved the gap junction intercellular communication (GJIC) function, and upregulated the proteins essential to gap junction, such as connexin 32 and connexin 43, indicating the potential role in enhancing the bystander effect of HSC-

TK/GCV. By co-culturing the B16 ${ }^{\mathrm{TK}}$ cells, which stably expressed TK gene, with wildtype B16 $\left(B 16^{\mathrm{WT}}\right.$ ) cells, we found that co-treatment of curcumin and GCV synergistically inhibited B16 cell proliferation, but the effect could be eliminated by the gap junction inhibitor AGA. Moreover, curcumin markedly increased apoptosis rate of B16 ${ }^{\mathrm{WT}}$ cells, suggesting its effect in enhancing the bystander effect of HSV-TK/GCV. In the in-vivo study, we established the xenografted melanoma model in 14 days by injecting mixture of $\mathrm{B} 16^{\mathrm{TK}}$ and $\mathrm{B} 16^{\mathrm{WT}}$ cell in a ratio of 3:7. The result demonstrated that, co-administration of curcumin and GCV significantly inhibited the xenograft growth, as indicated by the smaller size and less weight. The combinational effect was further confirmed as a synergistic effect. In conclusion, the results demonstrated that curcumin could enhance the killing effect and the bystander effect of HSV-TK/GCV in treating melanoma, which might be mediated by improved gap junction. Our data suggested that combination of HSV-TK/GCV with curcumin could be a potential chemosensitization strategy for cancer treatment. 


\section{Curcumin plays a synergistic role in combination with HSV-}

2 TK/GCV in inhibiting growth of murine B16 melanoma cells

3 and melanoma xenografts

4 Hong Li 1,2, *, Haiyan Du 1,2, *, Guangxian Zhang 1,2, Yingya Wu ${ }^{1}$, Pengxiang Qiu ${ }^{1}$, Jingjing

5 Liu ${ }^{3}$, Jing Guo ${ }^{1}$, Xijuan Liu ${ }^{1}$, Lingling Sun ${ }^{4}$, Biaoyan $\mathrm{Du}^{2,3}$, Yuhui Tan 1,2

$6{ }^{1}$ Department of Biochemistry and Molecular Biology, Guangzhou University of Chinese

7 Medicine, Guangzhou, China

82 The Research Center of Basic Integrative Medicine, Guangzhou University of Chinese

9 Medicine, Guangzhou, China;

$10{ }^{3}$ Department of Pathology, Guangzhou University of Chinese Medicine, Guangzhou, China.

$11{ }^{4}$ Integrative Cancer Center, First Affiliated Hospital, Guangzhou University of Chinese

12 Medicine, Guangzhou, China;

* These authors contributed equally to this work.

Corresponding Author:

Biaoyan Du; Yuhui Tan

Guangzhou University of Chinese Medicine (Higher Education Mega Center), 232 East Waihuan Rd., Guangzhou 510006, China.

Email address: dubiaoyan@gzucm.edu.cn (B.D.); yhuitan@foxmail.com (Y.T.).

\section{Abstract}

Melanoma is a global concern and accounts for the major mortality of skin cancers. Herpes simplex virus thymidine kinase gene with ganciclovir (HSV-TK/GCV) is a promising gene therapy for melanoma. Despite its low efficiency, it is well known for its bystander effect which is mainly mediated by gap junction. In this study, we found that curcumin reduced B16 melanoma cell viability in both time- and dose-dependent manner. Further study showed that curcumin improved the gap junction intercellular communication (GJIC) function, and upregulated the proteins essential to gap junction, such as connexin 32 and connexin 43, indicating the potential role in enhancing the bystander effect of HSC-TK/GCV. By co-culturing the B16 ${ }^{\mathrm{TK}}$ cells, which stably expressed TK gene, with wildtype B16 (B16 ${ }^{\mathrm{WT}}$ ) cells, we found that co-treatment of curcumin and GCV synergistically inhibited B16 cell proliferation, but the effect could be eliminated by the gap junction inhibitor AGA. Moreover, curcumin markedly increased apoptosis rate of B16 ${ }^{\mathrm{WT}}$ cells, suggesting its effect in enhancing the bystander effect of $\mathrm{HSV}-\mathrm{TK} / \mathrm{GCV}$. In the in-vivo study, we established the xenografted melanoma model in 14 days 
72

administration of curcumin and GCV significantly inhibited the xenograft growth, as indicated by the smaller size and less weight. The combinational effect was further confirmed as a synergistic effect. In conclusion, the results demonstrated that curcumin could enhance the killing effect and the bystander effect of HSV-TK/GCV in treating melanoma, which might be mediated by improved gap junction. Our data suggested that combination of HSV-TK/GCV with curcumin could be a potential chemosensitization strategy for cancer treatment.

\section{Introduction}

Melanoma is one of the cancers for which the incidence is still on the rise worldwide (Bray et al. 2018). Although melanoma only accounts for less than 5\% of skin cancers, it leads to the majority of deaths from skin cancers (Ferlay et al. 2019). Much attention has been attracted to improve the therapeutic strategy of this cancer (Prado et al. 2019; Wrobel et al. 2019). To date, for melanoma at early stage, surgical resection is a critical treatment. However, in most cases, it progresses into advanced stage and becomes resistant to conventional treatments (Heo et al. 2016). Hence, it is urgent to develop new therapies to counteract the limitation of conventional therapy and to slow down the pathological process.

During the last two decades, gene therapy has emerged as a promising alternative to treat a variety of human malignancies (Kumar et al. 2016). Suicide gene therapy is such a sort of gene therapy introducing the heterogeneous suicide gene that is able to converse a non-toxic prodrug into a lethal drug. Among various suicide systems, herpes simplex virus thymidine kinase gene with ganciclovir (HSV-TK/GCV) is one of the most in-depth-studied systems and has been introduced to treat melanoma (Navarro et al. 2016). In this system, viral thymidine kinase is expressed and subsequently metabolizes the prodrug GCV to mono-phosphorylated GCV, which will be further converted into triphosphate. Since GCV triphosphate is an analogue of deoxyguanosine triphostphate, it consequently inhibits DNA synthesis, causing the tumor cell death (Duarte et al. 2012). Of note, increasing evidence showed that the bystander effect, which is known to induce tumor regression despite that only a percentage of cancer cells express the TK gene, is a critical mechanism contributing to the anti-tumor effect of HSV-TK/GCV therapy (van Dillen et al. 2002). Since the efficiency still remains a challenge to HSV-TK/GCV therapy and it limits the clinical application, numerous studies have been focused on improving its bystander effect (Li et al. 2011; Rautsi et al. 2008; Xiao et al. 2018).

Curcumin is a bioactive component of Curcuma Longa L, which is a popular traditional medicine and culinary material in some countries (Ammon \& Wahl 1991). Due to its multiple biological properties, curcumin has been widely studied for its protective role in treating cancer, cardiovascular diseases, chronic inflammatory diseases, neurodegenerative diseases and arthritic (Aggarwal \& Harikumar 2009; Li et al. 2019; Willenbacher et al. 2019). Previous studies showed that curcumin treatment in melanoma cell lines or mice with melanoma xenograft 
79 demonstrated a growth-inhibited effect, and several cellular and molecular mechanisms have

80 been implicated (Mirzaei et al. 2016; Nabavi et al. 2018). However, whether curcumin has a

81 synergistic effect on HSV-TK/GCV therapy in melanoma still remains unknown. Therefore, in

82 the present study, we investigated the role of curcumin in B16 cells treated with HSV-TK/GCV

83 and its impact on the bystander effect, and further determined the synergistic effect in

84 xenografted melanoma.

85

86 Materials \& Methods

87 Cell culture

88 The murine malignant melanoma cell line B16 (wildtype, WT) was obtained from Sun Yat-Sen

89 University (Guangzhou, China). Cells were cultured in RPMI-1640 (Gibco/Invitrogen,

90 Burlington, Ontario, USA) supplemented with $10 \%$ fetal bovine serum (FBS) and $100 \mathrm{U} / \mathrm{mL}$

91 penicillin and streptomycin at $37^{\circ} \mathrm{C}$ in a humidified $5 \% \mathrm{CO}_{2}$ atmosphere. $\mathrm{B} 16^{\mathrm{TK}}$ cells, which are

92 B16 cells that stably express fused protein of HSV-TK and green fluorescent protein (GFP), and

$93 \mathrm{~B} 16^{\mathrm{WT}}$ cells that express red fluorescent protein (RFP) were generated in our lab. For curcumin

94 treatment, $20 \mathrm{mM}$ curcumin was freshly prepared in DMSO and then diluted into the desired

95 concentrations with the medium containing $10 \%$ FBS, followed by ultrasonication for $1 \mathrm{~min}$. The

96 dilutions were used to treat the cells immediately to avoid degradation.

97

98

99

100

101

102

103

104

105

106

107

108

109

110

111

112

113

114

115

116

117 Analysis of bystander Effect

\section{MTT assay}

Cells were seeded in 96-well plates at a density of 4000 cells per well and incubated overnight, followed by treated with various treatments. Then $10 \mu \mathrm{L}$ of $5 \mathrm{mg} / \mathrm{ml}$ MTT reagent (Sigma, St. Louis, MO, USA) was added to each well to incubate at $37^{\circ} \mathrm{C}$ for $4 \mathrm{~h}$, and the formazan crystals were dissolved in $150 \mu \mathrm{L}$ dimethyl sulphoxide (DMSO) in each well. Absorbance values were measured at wave length of $490 \mathrm{~nm}$ with a 96-well microplate reader (BioRad, Hercules, CA, USA). Each experiment was performed using 6 replicate wells for each treatment. The results were normalized to cells incubated in control medium, which were considered $100 \%$ viable.

\section{Double-fluorescence dye transfer assay}

B16 cells were treated with various concentrations of curcumin (Sigma, St. Louis, MO, USA) in duplicate for $48 \mathrm{~h}$. One population of cells in each group was stained with red fluorescein CMTMR and green fluorescein Calcein (Molecular Probes, Eugene, OR, USA) for $1 \mathrm{~h}$ at $37^{\circ} \mathrm{C}$. These cells were referred to "donor cells", while the other population of cells that was not subjected to fluorescein treatment was referred to "recipient cells". After washed with PBS, the donor cells and recipient cells were digested respectively and mixed at a ratio of 1:99. The mixed cells were seeded in dishes for $3 \mathrm{~h}$, and then digested for assessment by flow cytometry (BD Biosciences, San Jose, CA, USA). 
$118{\mathrm{~B} 16^{\mathrm{TK}} \text { cells and B16 }}^{\mathrm{WT}}$ cells were mixed in 1:4 and seeded in 6- or 96-well plates. $24 \mathrm{~h}$ later, the 119 cells were subjected to the following treatment, respectively: DMSO (negative control), GCV $120(15 \mu \mathrm{M})$, curcumin $(10 \mu \mathrm{M}$ or $20 \mu \mathrm{M})$, curcumin $(10 \mu \mathrm{M}$ or $20 \mu \mathrm{M})$ with $\mathrm{GCV}(15 \mu \mathrm{M})$. The cell

121

122

123

124

125

126

127

128

129

130

131

132

133

134

135

136

137

138

139

140

141

142

143

144

145

146

147

148

149

150

151

152

153

154

155

156

157

viability was detected by MTT assay. To determine the rate of apoptosis, the cells were digested and fixed in $70 \%$ ethanol at $4^{\circ} \mathrm{C}$, followed by stained with Annexin $\mathrm{V}$ for flow cytometry analysis. Of note, before harvested, pictures of cell morphology in each group were taken by fluorescence microscope.

\section{Western blot analysis}

B16 cells were cultured in 6-well plates for $48 \mathrm{~h}$, followed by treatment with curcumin $(5 \mu \mathrm{M}, 10$ $\mu \mathrm{M}, 20 \mu \mathrm{M}$ ) for another $24 \mathrm{~h}$. Then cells were homogenized in RIPA lysis buffer supplemented with protease inhibitor cocktail (Sigma, St. Louis, MO, USA) and $1 \mathrm{mM}$ PMSF. The lysates were incubated on ice for 30 minutes with a vortexing every 5 minutes, and centrifuged at 12,000 $\mathrm{g}$ for $30 \mathrm{~min}$. The supernatants were harvested, and then the protein concentrations were determined by BCA protein assay kit (Thermo Fisher Scientific, Rockford, Illinois, USA). An equal amount of the proteins $(30 \mu \mathrm{g})$ was subjected to $10 \%$ SDS-PAGE following the immunoblot procedure as demonstrated previously (Lu et al. 2016). Membranes were blocked with 5\% nonfat milk in TBS containing $0.1 \%$ Tween 20 for $90 \mathrm{~min}$, followed by incubation overnight at $4^{\circ} \mathrm{C}$ with specific antibodies to $\mathrm{Cx} 32$ (1:5000; ABconel, Wuhan, China), Cx43 (1:5000; ABconel, Wuhan, China) or GAPDH (1:10000, ABconel, Wuhan, China). Blots were developed with enhanced chemiluminescence HRP substrate (Millipore,Bedford, MA, USA) and detected by a Tanon detection system (Shanghai, China). The intensities of the blots were quantified by NIH image J software (Bio-Rad, Hercules, CA, USA).

\section{Animals}

SPF-class C57BL6/J mice (equivalent numbers of males and females, weighing 18-22 g) were supplied by the Experimental Animal Center of Sun Yat-Sen University (Guangzhou, China). All protocols were carried out in accordance with the Guide for the Care and Use of Laboratory Animals (NIH Publication No. 85-23, revised 1996), and were approved by the Institute Research Medical Ethics Committee of Guangzhou University of Chinese Medicine, China (20180408).

\section{Animal models of xenografted melanoma}

$\mathrm{B} 16^{\mathrm{TK}}$ cells and $\mathrm{B} 16^{\mathrm{WT}}$ cells were mixed at the indicated ratios and diluted with serum-free culture medium. A total of $2 \times 10^{5}$ cells in a final volume of $100 \mu \mathrm{L}$ was injected into the right flanks of each C57BL/6J mouse. The mice with tumor were randomized into four groups ( $\mathrm{n}=16$ mice per group): a saline control group, a group treated with GCV (50 mg/kg/day), a group treated with curcumin $(100 \mathrm{mg} / \mathrm{kg} /$ day $)$, and a group treated with both GCV $(50 \mathrm{mg} / \mathrm{kg} / \mathrm{day})$ and curcumin $(100 \mathrm{mg} / \mathrm{kg} /$ day $)$. For curcumin preparation, it was initially dissolved in DMSO and then diluted into $10 \mathrm{~g} / \mathrm{L}$ with saline solution, followed by ultrasonication for $1 \mathrm{~min}$ and 
158

159

160

161

162

163

164

165

166

167

168

169

170

171

172

173

174

175

176

177

178

179

180

181

182

183

184

185

186

187

188

189

190

191

192

193

194

195

196

197

administered immediately. Saline and curcumin were administratered intraperitoneally once a day during the study period of 14 days. GCV was administrated daily from day 7 till the end. Then the mice were sacrificed and the solid tumors were isolated to measure the volume and mass.

\section{Statistical analysis}

Data were presented as means \pm standard deviation (SD). By using SPSS 13.0, statistical analysis was performed with unpaired Student's $t$-test between two groups or one-way analysis of variance (ANOVA) followed by LSD test among various groups. In all cases, difference was considered statistically significant at $P<0.05$.

The combinational effect of two drugs was assessed by Q value using Zheng-Jun Jin's method (Jin 2004). Briefly, Q=EAB/[EA+EB (1-EA)], in which EA, EB and EAB represent the effect of drug $\mathrm{A}$, drug $\mathrm{B}$ and the combination of two drugs, respectively. According the $\mathrm{Q}$ value, the combinational effect can be deemed as an antagonistic effect ( $\mathrm{Q} \square 0.85)$, an additive effect $(0.85 \square \mathrm{Q} \square 1.15)$, or a synergistic effect $(\mathrm{Q} \square 1.15)$.

\section{Results}

1. Curcumin has a cytotoxicity effect on B16 cells.

Curcumin is a natural compound that has been delineated to be toxic to various cancer cells, but the activity varies from cell types and depends on the time of incubation. To determine the the optimal concentration of curcumin in combination with HSV-TK/GCV treatment, we conducted MTT assay. B16 cells were treated with 5, 10, 20, 40, and $80 \mu \mathrm{M}$ curcumin for $48 \mathrm{~h}$ or $72 \mathrm{~h}$. As shown in Fig. 1, $5 \mu \mathrm{M}$ cucumin was not cytotoxic, but higher concentration significantly inhibited B16 cells viability in both time- and dose-dependent manner. The half maximal inhibitory concentration (IC50) of curcumin in B16 cells was about $30 \mu \mathrm{M}$ at $48 \mathrm{~h}$ and $17.3 \mu \mathrm{M}$ at $72 \mathrm{~h}$, respectively. Notably, lower dose of curcumin $(5 \mu \mathrm{M}, 10 \mu \mathrm{M}$ and $20 \mu \mathrm{M})$ for $48 \mathrm{~h}$, which demonstrated relatively lower inhibitory effects on B16 cell viability, was used in other in-vitro studies to determine the effect of curcumin combined with HSV-TK/GCV treatment.

\section{Curcumin improved gap junction intercellular communication (GJIC) in B16 cells.} GJIC is a mode of cell-cell signaling that enables intercellular communication between adjacent cells through channels of connexins (Aasen et al. 2016), and plays a critical role in mediating the effect of drugs used in melanoma therapy (Aasen et al. 2019). To study whether curcumin affects the GJIC function of B16 cells, double-fluorescence dye transfer assay was carried out. In this study, the red fluorescent dye CMTMR and the green one Calcein were employed. Once entering into the cells, CMTMR retained in the living cells and could not be transferred to adjacent cells, whereas Calcein could be transferred between cells through GJ channel. In this sense, after double-dyed B16 cells were mixed with fluorescence-negative B16 cells by 1:99 and incubated for $4 \mathrm{~h}$, the ratio of cells with green fluorescence to those without fluorescence indicated the 
198

199

200

201

202

203

204

205

206

207

208

209

210

211

212

213

214

215

216

217

218

219

220

221

222

223

224

225

226

227

228

229

230

231

232

233

234

235

236

237

function of GJIC. As shown in Fig. 2 A and B, low dose of curcumin was sufficient to increase calcein transfer. Of note, all concentration tested significantly enhanced the function of GJIC, as indicated by the elevated calcein-positive cells compared to the control group. In addition, the influence of curcumin on connexins, which are essential to GJIC function, was also detected by Western Blot. We found that proteins of $\mathrm{Cx} 32$ and $\mathrm{Cx} 43$ were all upregulated by curcumin at the concentration of $20 \mu \mathrm{M}$ (Fig. $2 \mathrm{C}$ and $\mathrm{D}$ ).

\section{Curcumin synergistically improved the inhibitory effect of HSV-TV/GCV in B16 cells.} In order to study the effect of HSV-TK/GCV in B16 cells, we constructed B16 ${ }^{\mathrm{TK}}$ cells that stably expressed TK gene, and co-cultured these cells with B16 ${ }^{\mathrm{WT}}$ cells. As shown in Fig $3 \mathrm{~A}$, the antiproliferation effect of HSV-TK/GCV in B16 cells was dependent on the ratio of $\mathrm{B} 16^{\mathrm{TK}}$ cells to B16 ${ }^{\mathrm{WT}}$ cells. Since $20 \% \mathrm{~B} 16^{\mathrm{TK}}$ cells were sufficient to mediate the inhibitory effect of GCV, we co-cultured the $\mathrm{B} 16^{\mathrm{TK}}$ cells and $\mathrm{B} 16^{\mathrm{WT}}$ cells in 1:4 for the subsequent in-vitro studies. By using the MTT assay, we found that 5,10 and $20 \mu \mathrm{M}$ curcumin significantly improved the inhibitory effect of HSV-TK/GCV on B16 cell viability (Fig. 3 B). The combinational effect was scored by $\mathrm{Q}$ value, which was $1.85,1.43$ and 1.16 , respectively, indicating the synergistic effect of curcumin and HSV-TK/GCV treatment. Since curcumin inhibited GJIC function, we speculated that the synergistic effect might be mediated by gap junction. Hence, we examined whether the effect was inhibited by AGA, a long-term inhibitor of GJ. As demonstrated in Fig 3 C, AGA pretreatment evidently reversed the inhibitory effect of GCV combined with $5 \mu \mathrm{M}$ curcumin. The Q value was reduced from 1.34 to 0.68 , indicating an antagonistic effect (Jin 2004).

\section{The bystander effect of HSV-TK/GCV was enhanced by curcumin.}

The effect of suicide gene therapy is critically associated with its bystander effect (Duarte et al. 2012; van Dillen et al. 2002). To examine whether curcumin affected the bystander effect of HSV-TK/GCV treatment, we co-cultured the $\mathrm{B} 16^{\mathrm{TK}}$ cells and $\mathrm{B} 16^{\mathrm{WT}}$ cells at the ratio of 1:4. The B $16^{\mathrm{TK}}$ cells and B16 ${ }^{\mathrm{WT}}$ cells expressed GFP and RFP, respectively. Since GFP is fused with TK and that TK is a nucleoprotein, the B16 $6^{\mathrm{TK}}$ cells expressed GFP in the nuclear. By contrast, RFP in the $\mathrm{B} 16^{\mathrm{WT}}$ cells was mainly expressed in the cytoplasm. The fluorescence images showed that co-treatment of curcumin with GCV markedly induced B16 ${ }^{\mathrm{WT}}$ cells apoptosis, as indicated by pyknosis and cell shrinking. Subsequently, the apoptosis rate of B16 ${ }^{\mathrm{WT}}$ cells was detected by flow cytometry analysis. The result (Fig. 4 B) showed that, compared with the negative control, $20 \mu \mathrm{M}$ curcumin alone was able to induce B16 cell apoptosis, as indicated by the B16 ${ }^{\mathrm{WT}}$ cells stained with Annexin V. Interestingly, compared to the GCV group, the apoptosis rate was significantly elevated by combinational treatment with either 10 or $20 \mu \mathrm{M}$ curcumin, suggesting the potentiation of the bystander effect by curcumin. The $\mathrm{Q}$ value was 2.71 and 1.69 , respectively, which also suggested a synergistic effect.

\section{Combination of curcumin with HSV-TK/GCV therapy inhibited xenografted melanoma growth.}


238 To confirm the synergistic effect of curcumin on HSV-TK/GCV, we performed in vivo study 239 using the xenografted melanoma model (Fig. 5 A). In order to determine the optimal ratio of $240 \mathrm{~B} 16^{\mathrm{TK}}$ to B16 ${ }^{\mathrm{WT}}$ cells, we conducted preliminary experiment and injected the cell mixture with $24120 \%, 40 \%$, and $80 \% \mathrm{~B} 16^{\mathrm{TK}}$ cells, respectively, into the right flank of mice. 7 days later, GCV 242 was administrated for another 7-day period. Then the xenografted tumors were isolated. The 243 result showed that $\mathrm{B} 16^{\mathrm{TK}}$ cells at the percentage of $40 \%$ or $80 \%$ significantly reduced the tumor 244 weight (Fig. 5 B). To better evaluate the combinational effect of curcumin, we mixed B16 ${ }^{\mathrm{TK}}$ cells 245 with $\mathrm{B} 16^{\mathrm{WT}}$ cells in a ratio of 3:7 in the following in-vivo study. The result demonstrated that administration of neither GCV nor curcumin alone affected the size of the xenografted tumor (Fig. 5 C). By contrast, the size on day 12 and 13 was significantly reduced by co-administration of curcumin with GCV. On the $14^{\text {th }}$ day, the mice were sacrificed, and the tumor was isolated for measuring volume and mass. As shown in Fig. 5 D, while curcumin mildly influenced the weight

250

251

252

253

254

255

256

257

258

259

260

261

262

263

264

265

266

267

268

269

270

271

272

273

274

275

276

277

of xenografted melanoma, GCV exerted an evident inhibitory effect. Notably, combination of curcumin with GCV significantly inhibited the tumor growth, resulting in smaller size (Fig. 5 C) and less weight (Fig. $5 \mathrm{D}$ ). The $\mathrm{Q}$ value for the size (1.17) and weight (1.34) suggested that curcumin exerted a synergistic inhibitory effect with HSV-TK/GCV.

\section{Discussion}

Melanoma has been a global concern due to its high mortality and resistant to the conventional therapy such as chemotherapy and radiotherapy (Bray et al. 2018; Ferlay et al. 2019; Heo et al. 2016). Much effort has been made to improve the therapy strategy (Prado et al. 2019; Wrobel et al. 2019). As a promising therapeutic method, gene therapy has been widely introduced to treat melanoma and other tumors (Kumar et al. 2016; Sotomayor et al. 2002). However, due to the problem of low efficiency, which caused the insufficient lethality, it is limited in clinical application. Accordingly, using new delivery vectors or taking advantage of the combinational therapy has been a strategy to improve the therapy efficacy (Bressy et al. 2017; Luo et al. 2010; Vago et al. 2016).

HSV-TK/GCV is a well-known suicide gene therapy. In this system, viral thymidine kinase is expressed and consequently metabolizes GCV to mono-phosphorylated GCV. The product will be further phosphorylated by kinases into tri-phosphorylated GCV, an analogue of deoxyguanosine triphostphate, and thus inhibited DNA synthesis, resulting in the tumor cell death (Duarte et al. 2012). Of interest, in addition to the suicide effect of this system, the bystander effect was found to be particularly important to the antitumor activity (van Dillen et al. 2002). In this study, we constructed the $\mathrm{B} 16^{\mathrm{TK}}$ cells, which stably expressed the TK gene. To evaluate the effect of HSV-TK/GCV in B16 cells, we mixed B16 ${ }^{\mathrm{TK}}$ cells and B16 ${ }^{\mathrm{WT}}$ cells in different ratios. The result showed that $\mathrm{B} 16^{\mathrm{TK}}$ cells dose-dependently induced growth inhibition and bystander effect. Notably, 20\% B16 ${ }^{\mathrm{TK}}$ cells significantly inhibited the cell viability (Fig. 3 A), whereas the bystander effect was weak, as indicated by the less cell death than the supposed $20 \% \mathrm{~B} 16^{\mathrm{TK}}$ cells. Besides, increasing proportion of $\mathrm{B} 16^{\mathrm{TK}}$ cells in mixed cells injected into mice 
278 resulted in higher impact on tumor growth, as indicated by the xenograft weight (Fig. 5 A).

279 These results suggested that both inhibition effect and the bystander effect were influenced by

280 the transfection efficiency. Since the efficiency varies from vector and is always relatively low in

281 humans, we mixed B16 ${ }^{\mathrm{TK}}$ cells with B16 ${ }^{\mathrm{WT}}$ cells in 1:4 in the in-vitro study and 3:7 in the in-

282 vivo study, respectively, to study the synergistic effect of curcumin.

283

284

Since the bystander effect is critical to the killing action of HSV-TK/GCV therapy, it is

285 important to reveal the mechanism of the effect and more importantly, to seek ways to enhance

286

287 the effect. To date, several mechanisms have been demonstrated to mediate the bystander effect

288 of HSV-TK/GCV system, including the involvement of gap junction, E-cadherin regulation and soluble factors release (Asklund et al. 2003; Drake et al. 2000; Elshami et al. 1996; GarciaRodriguez et al. 2011). Our previous study showed that increased expression of $\mathrm{Cx} 43$, the most

290

291

292

293

294

295

296

297

298

299

300

301

302

303

304

305

306

307

308

309

310

311

312

313

314

315

316

317 predominant protein of gap junction, was critically involved in the therapeutic efficacy of melanoma (Kou et al. 2017; Xiao et al. 2018). In this study, we found that curcumin was able to induce connexins expression and elevate the GJIC function in B16 cells, indicating its potential activity to strengthen the bystander effect.

Curcumin is an active component of turmeric and is recognized as an antitoxic product in response to adverse stimuli such as UV radiation, mechanical injuries and fungi or virus infections (Ammon \& Wahl 1991). Recent decades have witnessed the multiple biological activities of curcumin in various diseases (Aggarwal \& Harikumar 2009), although some scientists deemed curcumin as a PAINS (pan-assay interference compounds) and an IMPS (invalid metabolic panaceas) because of its potential PAINS-type behaviors such as membrane disruption, aggregation and fluorescence interference (Nelson et al. 2017). Actually, the fact that curcumin was limited in clinical use might be mainly because of its poor solubility, low availability and instability (Aggarwal \& Harikumar 2009). However, much effect has been made to overcome this problem, and some stable and bioavailable curcumin formulations have been available on market, with scientifically proven benefits (Antony et al. 2008; Bahadori \& Demiray 2017; Belcaro et al. 2010; Morimoto et al. 2013).To date, accumulating studies showed that curcumin was effective in inhibiting melanoma cell proliferation and melanoma growth (Mirzaei et al. 2016; Nabavi et al. 2018; Zhang et al. 2015; Zhao et al. 2016). Consistently, we found that curcumin inhibited B16 cell viability in both time- and dose-dependent manner. Moreover, curcumin significantly enhanced the inhibitory effect of HSV-TK/GCV in B16 cell viability, and it was a synergistic effect as evaluated by Jin's method (Jin 2004). Of interest, the synergistic effect was abolished by the gap junction inhibitor AGA. However, since curcumin could interact with phospholipids (Shehzad et al. 2014) and membrane proteins (Ingolfsson et al. 2014) and that gap junction channels are localized at plasma membrane, it is possible that the strengthened effect of curcumin on GJIC might be mediated by some other potential effects of curcumin on membranes. Further study is still required to determine the specific mechanism by loss- and gain-of-function strategy.

Peer) reviewing PDF | (2019:06:38798:1:1:REVIEW 16 Aug 2019) 
318 Curcumin has been demonstrated to sensitize various types of cancer cells (Gopinath \& Ghosh 319 2008), in which it activates a set of apoptotic pathways, and thus exhibits the anti-proliferation 320 effect (Dorai et al. 2001; Huang et al. 1994; Radhakrishna Pillai et al. 2004). Our previous study 321 showed that HSV-TK/GCV treatment induced cell apoptosis and inhibited tumor growth (Xiao et 322 al. 2018). By loading curcumin into the P7L10 peptide micelles to be a more efficient carrier for 323 HSV-TK gene delivery, Park et al. reported that HSV-tk/R7L10-Cur complex induced C6 rat

324

325

326

327

328

329

330

331

332

333

334

335

336

337

338

339

340

341

342

343

344

345

346

347

348

349

350

351

352

353

354

355

356

357

358 glioblastoma cell death and reduced the tumor size of xenografted glioblastoma (Park et al. 2015). However, the mechanism was not studied and the dose of curcumin was not demonstrated. In this study, we found that B16 cells co-treated with curcumin and GCV exhibited significantly higher apoptosis rate, and the bystander effect of HSV-TK/GCV was also enhanced by curcumin, as indicated by increased apoptotic B16 ${ }^{\mathrm{WT}}$ cells. The combinational effect was further confirmed in the in-vivo study. Consistently, curcumin synergistically strengthened the anticancer effect of HSV-TK/GCV, resulting in reduced xenografted melanoma size and mass. Of note, the inhibitory effect of curcumin or GCV alone on the xenografted melanoma was not statistically significant in this study, which might be attributed to the low proportion of $\mathrm{B} 16^{\mathrm{TK}}$ cells and low bioavailability or instability of curcumin, respectively.

\section{Conclusions}

In summary, the chemotherapeutic effect of HSV-TK/GCV treatment is delineated using vectorbased expression of TK in cancer cells, where it converts the prodrug GCV to lethal GCV triphosphate (Duarte et al. 2012). The bystander effect that neighboring cells, without TK expression, also happen to die is essential to its anti-cancer effects (van Dillen et al. 2002). Curcumin is a natural compound that possesses diverse activities (Prasad et al. 2014). In the present study, we constructed $\mathrm{B} 16^{\mathrm{TK}}$ cells stably expressed TK gene, and mixed with B16 ${ }^{\mathrm{WT}}$ cells to study the bystander effects of HSV-TK/GCV, as well as the combinational role of curcumin. Our data disclosed that curcumin synergistically enhanced the anti-proliferation and bystander effects of HSV-TK/GCV. Combinational treatment of curcumin and HSV-TK/GCV effectively inhibited xenografted melanoma growth. These findings provide new insight into the combination therapy of curcumin and HSV-TK/GCV, and may provide a novel therapeutic strategy to increase gene therapy efficacy.

\section{References}

Aasen T, Leithe E, Graham SV, Kameritsch P, Mayan MD, Mesnil M, Pogoda K, and Tabernero A. 2019. Connexins in cancer: bridging the gap to the clinic. Oncogene. 10.1038/s41388019-0741-6

Aasen T, Mesnil M, Naus CC, Lampe PD, and Laird DW. 2016. Gap junctions and cancer: communicating for 50 years. Nat Rev Cancer 16:775-788. 10.1038/nrc.2016.105

Aggarwal BB, and Harikumar KB. 2009. Potential therapeutic effects of curcumin, the antiinflammatory agent, against neurodegenerative, cardiovascular, pulmonary, metabolic, autoimmune and neoplastic diseases. Int J Biochem Cell Biol 41:40-59. 10.1016/j.biocel.2008.06.010 
359

360

361

362

363

364

365

366

367

368

369

370

371

372

373

374

375

376

377

378

379

380

381

382

383

384

385

386

387

388

389

390

391

392

393

394

395

396

397

398

399

400

401

402
Ammon HP, and Wahl MA. 1991. Pharmacology of Curcuma longa. Planta Med 57:1-7. $10.1055 / \mathrm{s}-2006-960004$

Antony B, Merina B, Iyer VS, Judy N, Lennertz K, and Joyal S. 2008. A Pilot Cross-Over Study to Evaluate Human Oral Bioavailability of BCM-95CG (Biocurcumax), A Novel Bioenhanced Preparation of Curcumin. Indian J Pharm Sci 70:445-449. 10.4103/0250474x.44591

Asklund T, Appelskog IB, Ammerpohl O, Langmoen IA, Dilber MS, Aints A, Ekstrom TJ, and Almqvist PM. 2003. Gap junction-mediated bystander effect in primary cultures of human malignant gliomas with recombinant expression of the HSVtk gene. Exp Cell Res 284:185-195.

Bahadori F, and Demiray M. 2017. A Realistic View on "The Essential Medicinal Chemistry of Curcumin". ACS Med Chem Lett 8:893-896. 10.1021/acsmedchemlett.7b00284

Belcaro G, Cesarone MR, Dugall M, Pellegrini L, Ledda A, Grossi MG, Togni S, and Appendino G. 2010. Efficacy and safety of Meriva(R), a curcumin-phosphatidylcholine complex, during extended administration in osteoarthritis patients. Altern Med Rev 15:337-344.

Bray F, Ferlay J, Soerjomataram I, Siegel RL, Torre LA, and Jemal A. 2018. Global cancer statistics 2018: GLOBOCAN estimates of incidence and mortality worldwide for 36 cancers in 185 countries. CA Cancer J Clin 68:394-424. 10.3322/caac.21492

Bressy C, Hastie E, and Grdzelishvili VZ. 2017. Combining Oncolytic Virotherapy with p53 Tumor Suppressor Gene Therapy. Mol Ther Oncolytics 5:20-40. 10.1016/j.omto.2017.03.002

Dorai T, Cao YC, Dorai B, Buttyan R, and Katz AE. 2001. Therapeutic potential of curcumin in human prostate cancer. III. Curcumin inhibits proliferation, induces apoptosis, and inhibits angiogenesis of LNCaP prostate cancer cells in vivo. Prostate 47:293-303. 10.1002/pros. 1074

Drake RR, Pitlyk K, McMasters RA, Mercer KE, Young H, and Moyer MP. 2000. Connexinindependent ganciclovir-mediated killing conferred on bystander effect-resistant cell lines by a herpes simplex virus-thymidine kinase-expressing colon cell line. Mol Ther 2:515523. 10.1006/mthe.2000.0192

Duarte S, Carle G, Faneca H, de Lima MC, and Pierrefite-Carle V. 2012. Suicide gene therapy in cancer: where do we stand now? Cancer Lett 324:160-170. 10.1016/j.canlet.2012.05.023

Elshami AA, Saavedra A, Zhang H, Kucharczuk JC, Spray DC, Fishman GI, Amin KM, Kaiser LR, and Albelda SM. 1996. Gap junctions play a role in the 'bystander effect' of the herpes simplex virus thymidine kinase/ganciclovir system in vitro. Gene Ther 3:85-92.

Ferlay J, Colombet M, Soerjomataram I, Mathers C, Parkin DM, Pineros M, Znaor A, and Bray F. 2019. Estimating the global cancer incidence and mortality in 2018: GLOBOCAN sources and methods. Int J Cancer 144:1941-1953. 10.1002/ijc.31937

Garcia-Rodriguez L, Abate-Daga D, Rojas A, Gonzalez JR, and Fillat C. 2011. E-cadherin contributes to the bystander effect of TK/GCV suicide therapy and enhances its antitumoral activity in pancreatic cancer models. Gene Ther 18:73-81. $10.1038 /$ gt.2010.114

Gopinath P, and Ghosh SS. 2008. Apoptotic induction with bifunctional E.coli cytosine deaminase-uracil phosphoribosyltransferase mediated suicide gene therapy is synergized by curcumin treatment in vitro. Mol Biotechnol 39:39-48. 10.1007/s12033-007-9026-3

PeerJ reviewing PDF | (2019:06:38798:1:1:REVIEW 16 Aug 2019) 
403

404

405

406

407

408

409

410

411

412

413

414

415

416

417

418

419

420

421

422

423

424

425

426

427

428

429

430

431

432

433

434

435

436

437

438

439

440

441

442

443

444

445

446

447

448

Heo JR, Kim NH, Cho J, and Choi KC. 2016. Current treatments for advanced melanoma and introduction of a promising novel gene therapy for melanoma (Review). Oncol Rep 36:1779-1786. 10.3892/or.2016.5032

Huang MT, Lou YR, Ma W, Newmark HL, Reuhl KR, and Conney AH. 1994. Inhibitory effects of dietary curcumin on forestomach, duodenal, and colon carcinogenesis in mice. Cancer Res 54:5841-5847.

Ingolfsson HI, Thakur P, Herold KF, Hobart EA, Ramsey NB, Periole X, de Jong DH, Zwama M, Yilmaz D, Hall K, Maretzky T, Hemmings HC, Jr., Blobel C, Marrink SJ, Kocer A, Sack JT, and Andersen OS. 2014. Phytochemicals perturb membranes and promiscuously alter protein function. ACS Chem Biol 9:1788-1798. 10.1021/cb500086e

Jin ZJ. 2004. About the evaluation of drug combination. Acta Pharmacol Sin 25:146-147.

Kou Y, Ji L, Wang H, Wang W, Zheng H, Zou J, Liu L, Qi X, Liu Z, Du B, and Lu L. 2017. Connexin 43 upregulation by dioscin inhibits melanoma progression via suppressing malignancy and inducing M1 polarization. Int J Cancer 141:1690-1703. $10.1002 / \mathrm{ijc} .30872$

Kumar SR, Markusic DM, Biswas M, High KA, and Herzog RW. 2016. Clinical development of gene therapy: results and lessons from recent successes. Mol Ther Methods Clin Dev 3:16034. 10.1038/mtm.2016.34

Li H, Sureda A, Devkota HP, Pittala V, Barreca D, Silva AS, Tewari D, Xu S, and Nabavi SM. 2019. Curcumin, the golden spice in treating cardiovascular diseases. Biotechnol Adv. 10.1016/j.biotechadv.2019.01.010

Li S, Gao Y, Pu K, Ma L, Song X, and Liu Y. 2011. All-trans retinoic acid enhances bystander effect of suicide-gene therapy against medulloblastomas. Neurosci Lett 503:115-119. 10.1016/j.neulet.2011.08.019

Lu J, Sun D, Liu Z, Li M, Hong H, Liu C, Gao S, Li H, Cai Y, Chen S, Li Z, Ye J, and Liu P. 2016. SIRT6 suppresses isoproterenol-induced cardiac hypertrophy through activation of autophagy. Transl Res 172:96-112.e116. 10.1016/j.trs1.2016.03.002

Luo S, Chen P, Luo ZC, Zhang P, Sun P, Shi W, Li ZY, Zhang XL, Wang LQ, Chen X, Wei YQ, and Wen YJ. 2010. Combination of vesicular stomatitis virus matrix protein gene therapy with low-dose cisplatin improves therapeutic efficacy against murine melonoma. Cancer Sci 101:1219-1225. 10.1111/j.1349-7006.2010.01507.x

Mirzaei H, Naseri G, Rezaee R, Mohammadi M, Banikazemi Z, Mirzaei HR, Salehi H, Peyvandi M, Pawelek JM, and Sahebkar A. 2016. Curcumin: A new candidate for melanoma therapy? Int J Cancer 139:1683-1695. 10.1002/ijc.30224

Morimoto T, Sunagawa Y, Katanasaka Y, Hirano S, Namiki M, Watanabe Y, Suzuki H, Doi O, Suzuki K, Yamauchi M, Yokoji T, Miyoshi-Morimoto E, Otsuka Y, Hamada T, Imaizumi A, Nonaka Y, Fuwa T, Teramoto T, Kakeya H, Wada H, and Hasegawa K. 2013. Drinkable preparation of Theracurmin exhibits high absorption efficiency--a single-dose, double-blind, 4-way crossover study. Biol Pharm Bull 36:1708-1714. 10.1248/bpb.b13-00150

Nabavi SM, Russo GL, Tedesco I, Daglia M, Orhan IE, Nabavi SF, Bishayee A, Nagulapalli Venkata KC, Abdollahi M, and Hajheydari Z. 2018. Curcumin and Melanoma: From Chemistry to Medicine. Nutr Cancer 70:164-175. 10.1080/01635581.2018.1412485

Navarro SA, Carrillo E, Grinan-Lison C, Martin A, Peran M, Marchal JA, and Boulaiz H. 2016. Cancer suicide gene therapy: a patent review. Expert Opin Ther Pat 26:1095-1104. 10.1080/13543776.2016.1211640

Peer) reviewing PDF | (2019:06:38798:1:1:REVIEW 16 Aug 2019) 
449

450

451

452

453

454

455

456

457

458

459

460

461

462

463

464

465

466

467

468

469

470

471

472

473

474

475

476

477

478

479

480

481

482

483

484

485

486

487

488

489

490

Nelson KM, Dahlin JL, Bisson J, Graham J, Pauli GF, and Walters MA. 2017. The Essential Medicinal Chemistry of Curcumin. J Med Chem 60:1620-1637.

10.1021/acs.jmedchem.6b00975

Park JH, Han J, and Lee M. 2015. Thymidine kinase gene delivery using curcumin loaded peptide micelles as a combination therapy for glioblastoma. Pharm Res 32:528-537. 10.1007/s11095-014-1482-4

Prado G, Svoboda RM, and Rigel DS. 2019. What's New in Melanoma. Dermatol Clin 37:159168. 10.1016/j.det.2018.12.005

Prasad S, Gupta SC, Tyagi AK, and Aggarwal BB. 2014. Curcumin, a component of golden spice: from bedside to bench and back. Biotechnol Adv 32:1053-1064. 10.1016/j.biotechadv.2014.04.004

Radhakrishna Pillai G, Srivastava AS, Hassanein TI, Chauhan DP, and Carrier E. 2004. Induction of apoptosis in human lung cancer cells by curcumin. Cancer Lett 208:163170. 10.1016/j.canlet.2004.01.008

Rautsi O, Lehmusvaara S, Ketola A, Maatta AM, Wahlfors J, and Pellinen R. 2008. Characterization of HIV-1 TAT peptide as an enhancer of HSV-TK/GCV cancer gene therapy. Cancer Gene Ther 15:303-314. 10.1038/cgt.2008.17

Shehzad A, Ul-Islam M, Wahid F, and Lee YS. 2014. Multifunctional polymeric nanocurcumin for cancer therapy. J Nanosci Nanotechnol 14:803-814. 10.1166/jnn.2014.9103

Sotomayor MG, Yu H, Antonia S, Sotomayor EM, and Pardoll DM. 2002. Advances in gene therapy for malignant melanoma. Cancer Control 9:39-48. $10.1177 / 107327480200900106$

Vago R, Collico V, Zuppone S, Prosperi D, and Colombo M. 2016. Nanoparticle-mediated delivery of suicide genes in cancer therapy. Pharmacol Res 111:619-641. 10.1016/j.phrs.2016.07.007

van Dillen IJ, Mulder NH, Vaalburg W, de Vries EF, and Hospers GA. 2002. Influence of the bystander effect on HSV-tk/GCV gene therapy. A review. Curr Gene Ther 2:307-322.

Willenbacher E, Khan SZ, Mujica SCA, Trapani D, Hussain S, Wolf D, Willenbacher W, Spizzo G, and Seeber A. 2019. Curcumin: New Insights into an Ancient Ingredient against Cancer. Int J Mol Sci 20. 10.3390/ijms20081808

Wrobel S, Przybylo M, and Stepien E. 2019. The Clinical Trial Landscape for Melanoma Therapies. J Clin Med 8. 10.3390/jcm8030368

Xiao J, Wang X, Wu Y, Zhao Q, Liu X, Zhang G, Zhao Z, Ning Y, Wang K, Tan Y, and Du B. 2018. Synergistic effect of resveratrol and HSV-TK/GCV therapy on murine hepatoma cells. Cancer Biol Ther:1-9. 10.1080/15384047.2018.1523094

Zhang YP, Li YQ, Lv YT, and Wang JM. 2015. Effect of curcumin on the proliferation, apoptosis, migration, and invasion of human melanoma A375 cells. Genet Mol Res 14:1056-1067. 10.4238/2015.February.6.9

Zhao G, Han X, Zheng S, Li Z, Sha Y, Ni J, Sun Z, Qiao S, and Song Z. 2016. Curcumin induces autophagy, inhibits proliferation and invasion by downregulating AKT/mTOR signaling pathway in human melanoma cells. Oncol Rep 35:1065-1074. 10.3892/or.2015.4413

Peer] reviewing PDF | (2019:06:38798:1:1:REVIEW 16 Aug 2019) 
Figure 1

Fig. 1 Curcumin has a cytotoxicity effect on B16 cells.

B16 cells were treated with or without curcumin ( $5 \mu \mathrm{M}, 10 \mu \mathrm{M}, 20 \mu \mathrm{M}, 40 \mu \mathrm{M}$ and $80 \mu \mathrm{M})$, and then the cell viability at $48 \mathrm{~h}(\mathrm{~A})$ and $72 \mathrm{~h}$ (B) was determined by MTT assay, respectively. Data were presented as means $\pm \mathrm{SD}$. $* * P<0.01$ vs. ctrl group.

A

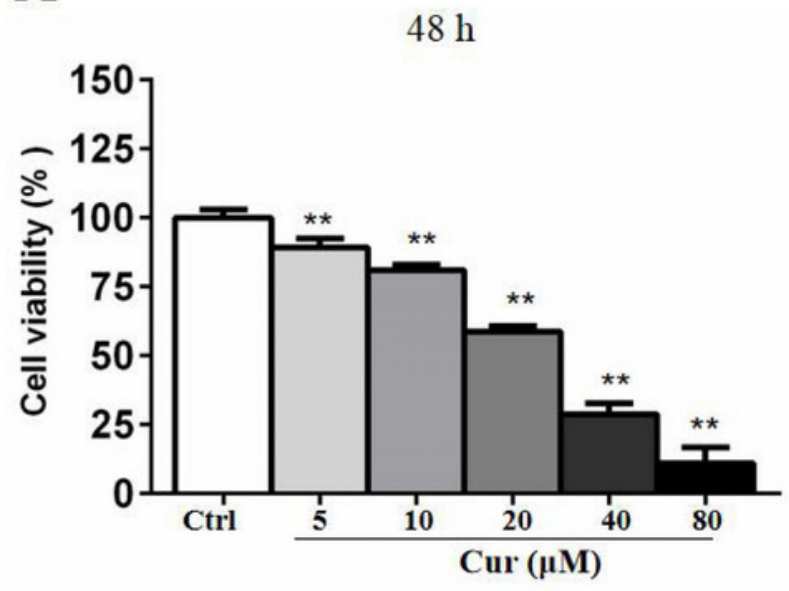

B

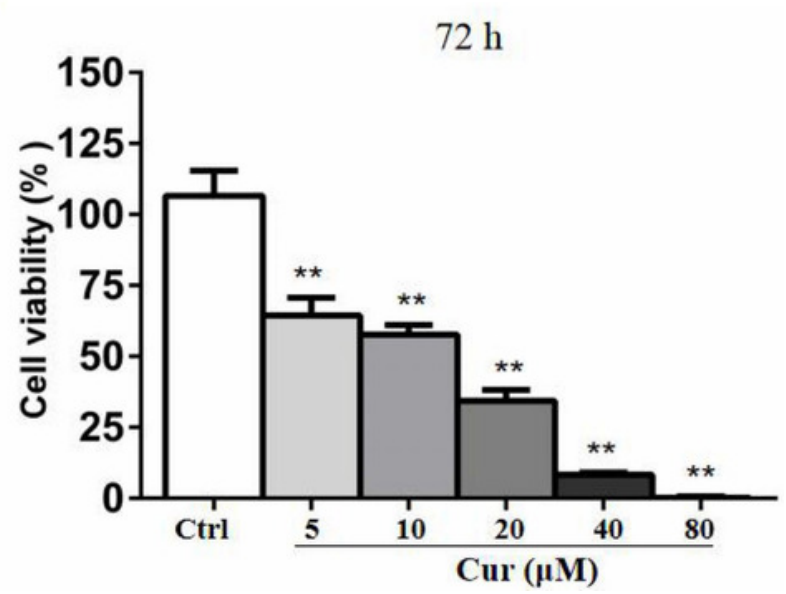




\section{Figure 2}

Fig. 2 Curcumin improved GJIC function in B16 cells.

Double-fluorescence dye transfer assay was used to evaluate GJIC function in B16 cells. (A)

Cells were sorted by flow cytometry. A1: calcein and $\mathrm{CMTMR}^{+}$cells, $\mathrm{A2}^{-}$: calcein ${ }^{+}$and $\mathrm{CMTMR}^{+}$ donor cells, A3: calcein and CMTMR recipient cells, A4: CMTMR and calcein ${ }^{+}$cells. (B) The proportion of CMTMR and calcein ${ }^{+}$cells in A4 quadrant was quantified. In the parallel study, B16 cells were subjected to curcumin treatment with indicated concentrations for $24 \mathrm{~h}$, followed by harvested for Western Blot analysis. Protein levels of $\mathrm{C} \times 32$ and $\mathrm{C} \times 43$ were detected (C) and quantified (D). Data were presented as means \pm SD. $* P<0.05$, $* * P<0.01$ vs. ctrl group.

$\mathbf{A}$
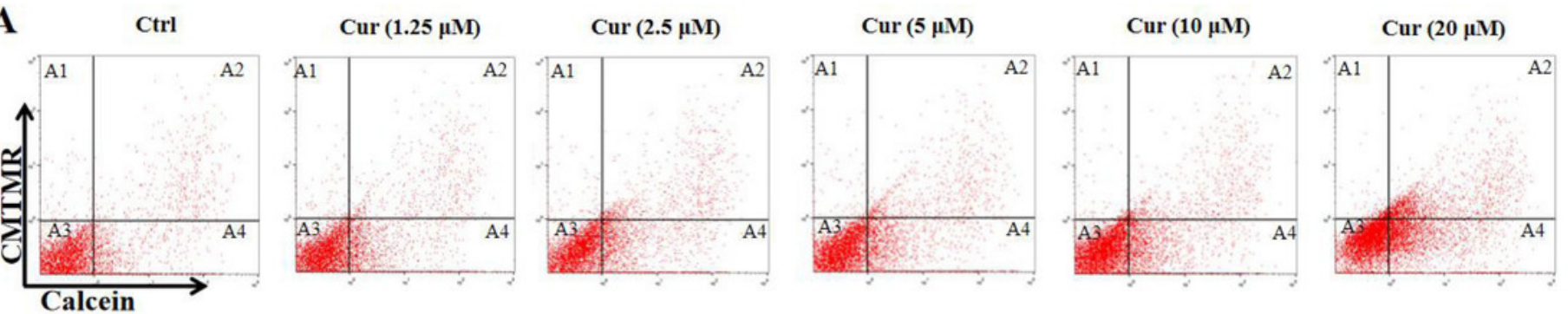

B

C

D
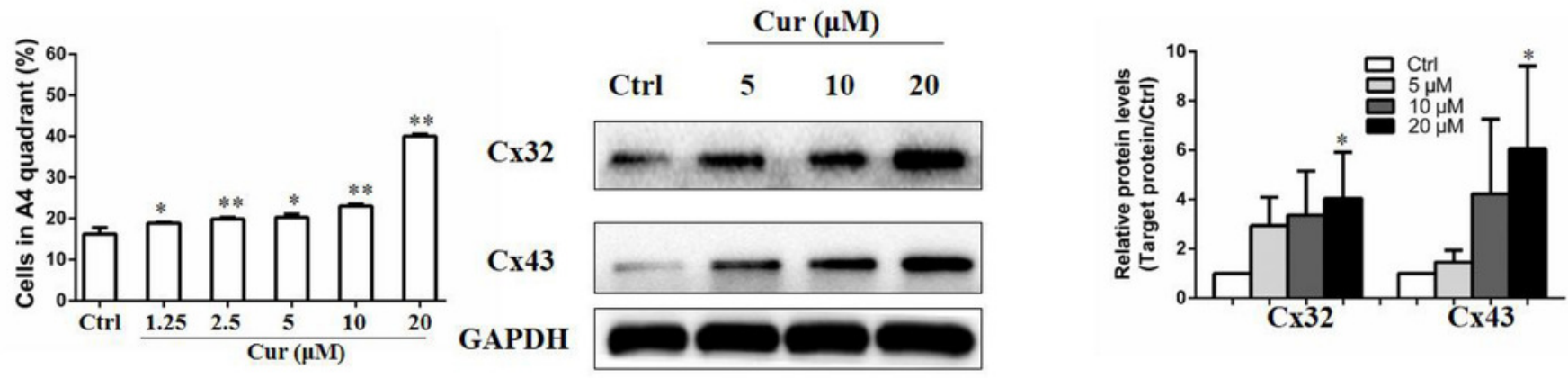


\section{Figure 3}

Fig. 3 Combination of curcumin and HSV-TK/GCV treatment had a synergistic inhibitory effect on the growth of B16 cells.

(A) $\mathrm{B} 16^{\mathrm{WT}}$ cells were co-cultured with indicated proportions of $\mathrm{B} 16^{\mathrm{TK}}$ cells in 96-well plates, followed by treatment with or without $15 \mu \mathrm{M} \mathrm{GCV}$ for $48 \mathrm{~h}$. The cell viability was determined by MTT assay. ${ }^{* * P}<0.01$ vs. GCV ctrl group. (B) $\mathrm{B}^{-} 6^{\mathrm{TK}}$ and $\mathrm{B}^{-\mathrm{WT}}$ cells were co-cultured in a ratio of 1:4 in 96-well plates. 24 h later, cells were pretreated with indicated concentrations of curcumin for $1 \mathrm{~h}$, followed by treatment with $15 \mu \mathrm{M}$ GCV for additional $48 \mathrm{~h}$. The cell viability

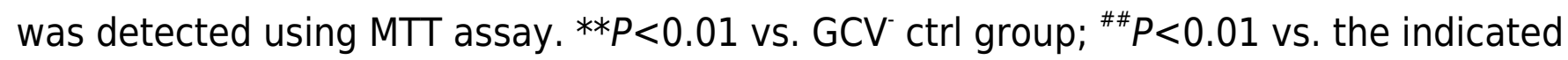
groups. (C) AGA treatment (15 $\mu \mathrm{M})$ impaired the inhibitory effect of GCV combined with $5 \mu \mathrm{M}$ curcumin on the mixed cells. ${ }^{* *} P<0.01$ vs. ctrl group; ${ }^{*} P<0.05$ vs. the indicated groups. Data were presented as means \pm SD. 
$\mathbf{A}$

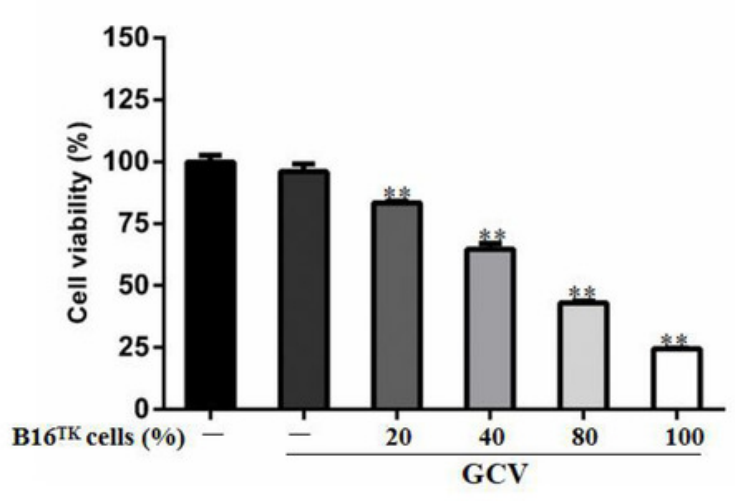

C

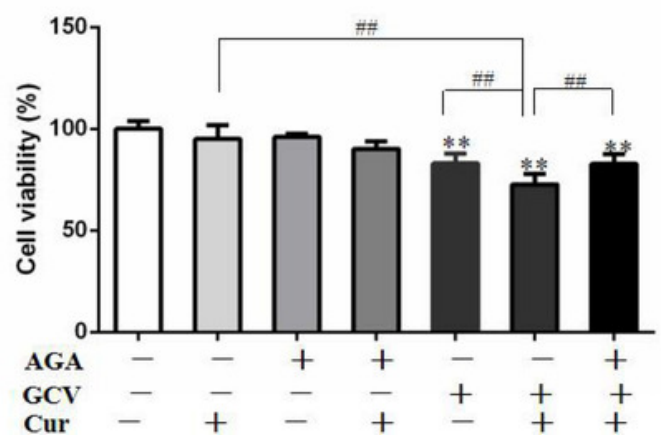

B

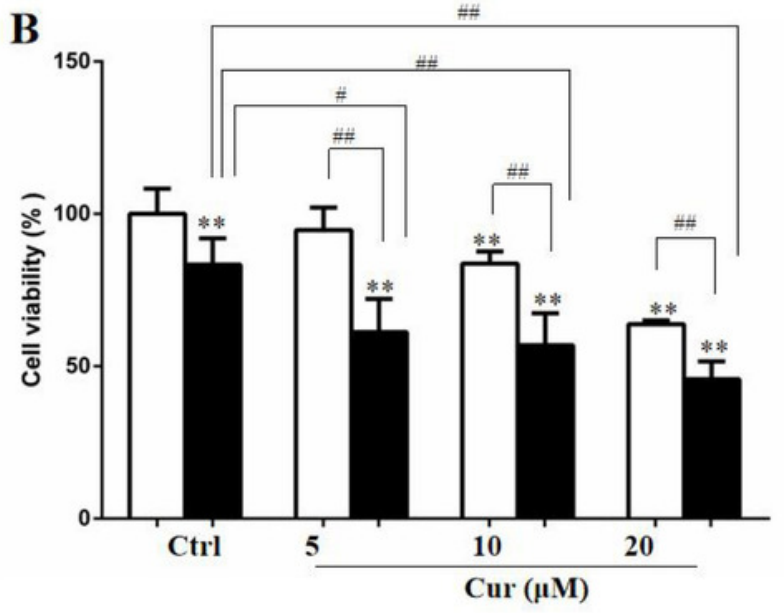

口 GCV

$\mathrm{GCV}^{+}$ 


\section{Figure 4}

Fig. 4 The bystander effect of HSV-TK/GCV was enhanced by curcumin.

$\mathrm{B}^{\mathrm{TK}}$ and $\mathrm{B} 16^{\mathrm{WT}}$ cells were mixed in 1:4 and cultured in a 6 -well plate. Cells were pretreated with or without indicated concentration of curcumin for $1 \mathrm{~h}$, and then exposed to $15 \mu \mathrm{M} \mathrm{GCV}$ for $72 \mathrm{~h}$. Cells were harvested and stained with Annexin $\mathrm{V}$ for detecting the apoptosis rate.

(A) GFP fused with TK B16 ${ }^{\text {TK }}$ cells and RFP in $B 16^{\text {WT }}$ cells were tracked and images were taken by fluorescence microscopy. The red color was converted into magenta (purple) for better accessibility. White arrows indicate the apoptotic cells. (B) The apoptosis rate of B16 ${ }^{\mathrm{WT}}$ cells.

Data were presented as means \pm SD. ${ }^{* *} P<0.01$ vs. GCV ctrl group; ${ }^{*} P<0.05,{ }^{\# \#} P<0.01$ vs. the indicated groups. 
A
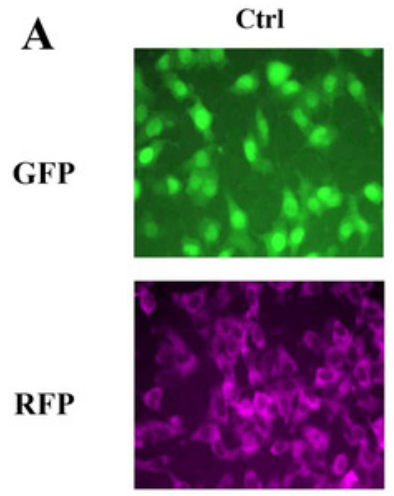

RFP

GFP

Merged

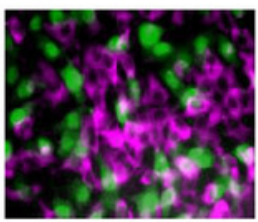

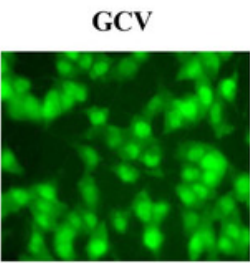
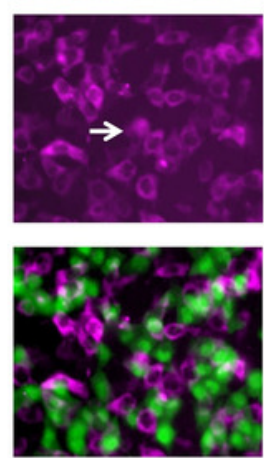
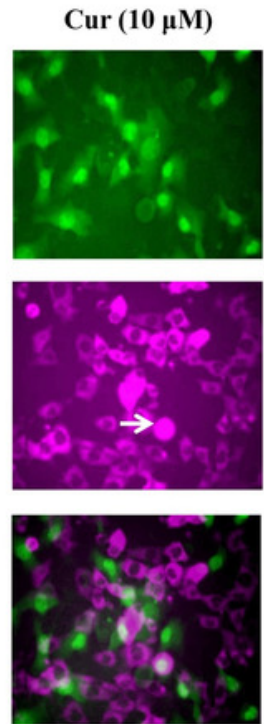

Cur $(10 \mu \mathrm{M})+\mathrm{GCV}$
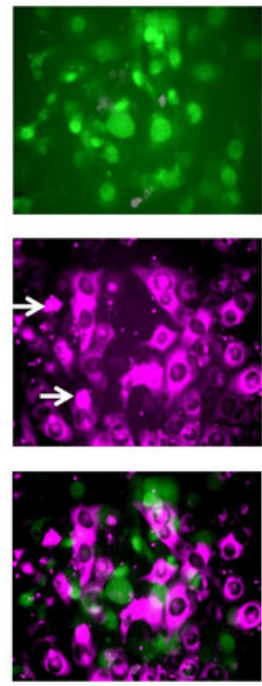
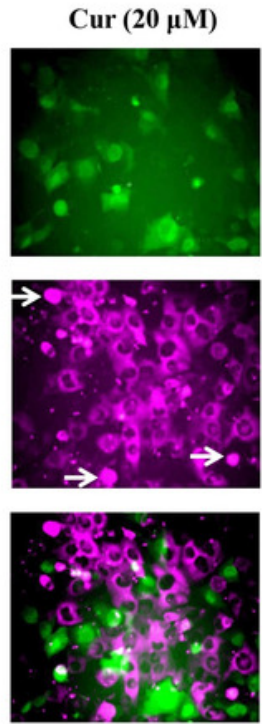

$\operatorname{Cur}(20 \mu \mathrm{M})+\mathrm{GCV}$
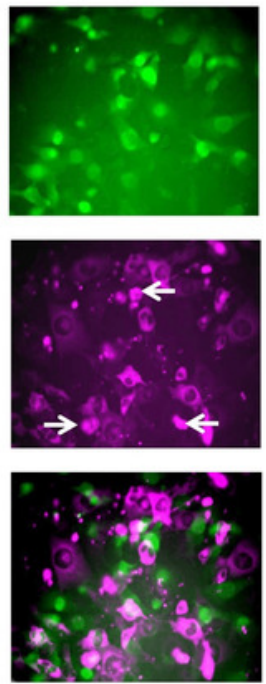

B

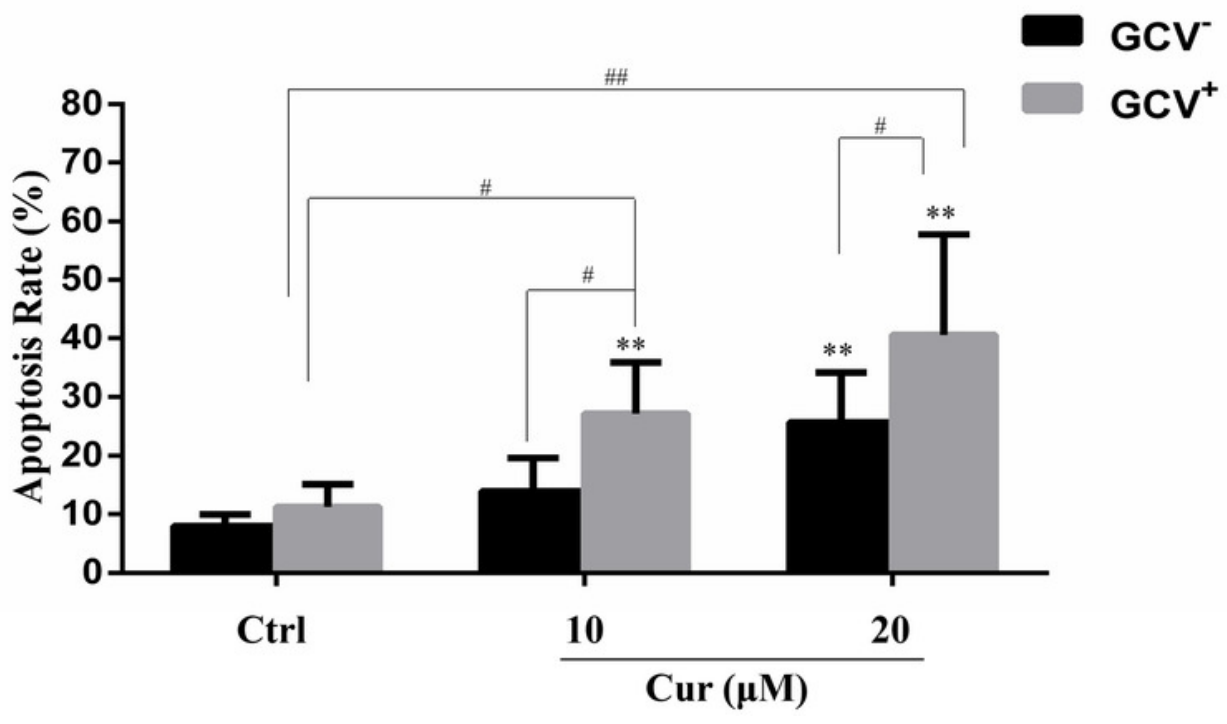




\section{Figure 5}

Fig. 5 Combination of curcumin with HSV-TK/GCV therapy enhanced the inhibitory effect on xenografted melanoma.

(A) Timeline of the in-vivo study. (B) B16 ${ }^{\text {TK }}$ and $\mathrm{B} 16^{\mathrm{WT}}$ cell mixture with $20 \%, 40 \%$, and $80 \%$ of B16 ${ }^{\text {TK }}$ cells was injected into the right flanks of each C57BL/6J mouse, followed by GCV administration. Then the xenografted tumors were isolated and weighed for analysis. $* P<0.05, * * P<0.01$ vs. the ctrl group. (C-D) Subcutaneous tumors were induced in C57BL/6) mice using $B 16^{\mathrm{TK}}$ and $B 16^{\mathrm{WT}}$ cells mixed at a ratio of 3:7. The mice were divided into 4 groups randomly, and subjected to the indicated treatments. (C) Tumor volume was measured daily since the $11^{\text {th }}$ day till the end. ${ }^{\$ \$} P<0.01$ vs. the ctrl group; $* P<0.05$, Cur+GCV group vs. Cur group; ${ }^{\#} P<0.05,{ }^{\# \#} P<0.01$, Cur+GCV group vs. GCV group. (D) The tumor weight of xenografted tumor. $* P<0.05, * * P<0.01$ vs. the indicated groups. Data were presented as means $\pm S D$. 
A $2 \times 10^{5}$ cells $/ \mathrm{ml}$ injection

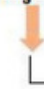

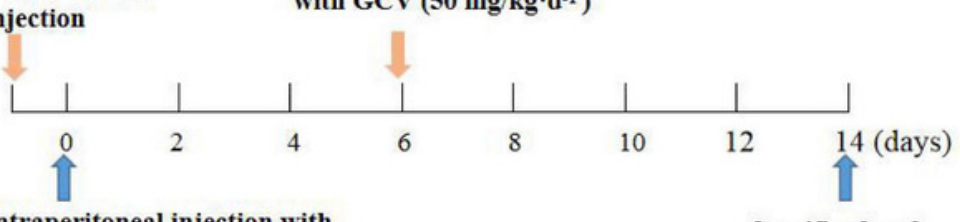

Intraperitoneal injection with

curcumin $\left(100 \mathrm{mg} / \mathrm{kg} \cdot \mathrm{d}^{-1}\right)$

Intraperitoneal injection with GCV (50 $\left.\mathrm{mg} / \mathrm{kg} \cdot \mathrm{d}^{-1}\right)$

Sacrificed and tissue isolation

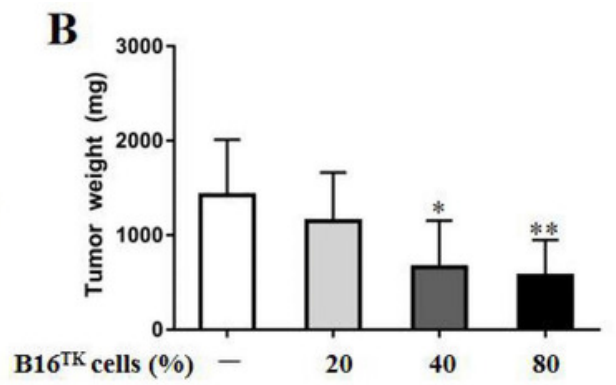

C

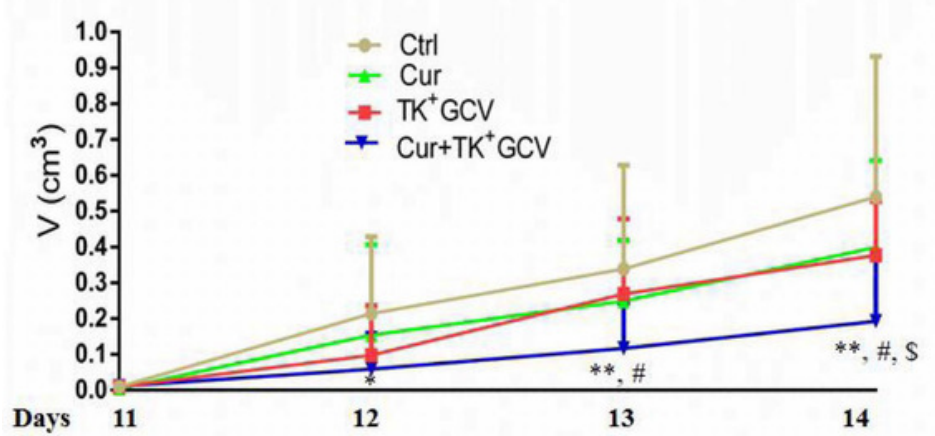

D

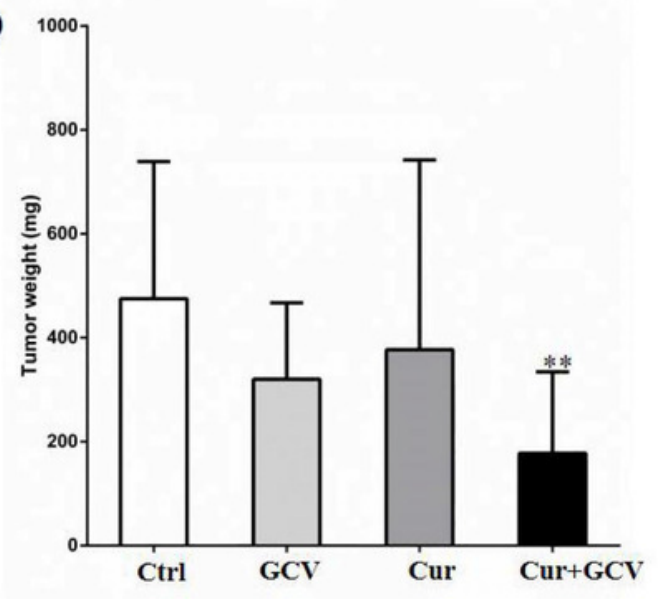

